\title{
Probing turbulence in the Coma galaxy cluster ${ }^{\star}$
}

\author{
P. Schuecker ${ }^{1}$, A. Finoguenov ${ }^{1}$, F. Miniati ${ }^{2}$, H. Böhringer ${ }^{1}$, and U. G. Briel ${ }^{1}$ \\ 1 Max-Planck-Institut für extraterrestrische Physik, Giessenbachstraße, 85741 Garching, Germany \\ e-mail: peters@mpe.mpg.de \\ 2 Max-Planck-Institut für Astrophysik, Karl-Schwarzschild-Str., 85741 Garching, Germany
}

Received 6 April 2004 / Accepted 8 July 2004

\begin{abstract}
Spatially-resolved gas pressure maps of the Coma galaxy cluster are obtained from a mosaic of XMM-Newton observations in the scale range between a resolution of $20 \mathrm{kpc}$ and an extent of $2.8 \mathrm{Mpc}$. A Fourier analysis of the data reveals the presence of a scale-invariant pressure fluctuation spectrum in the range between 40 and $90 \mathrm{kpc}$ and is found to be well described by a projected Kolmogorov/Oboukhov-type turbulence spectrum. Deprojection and integration of the spectrum yields the lower limit of $\sim 10$ percent of the total intracluster medium pressure in turbulent form. The results also provide observational constraints on the viscosity of the gas.
\end{abstract}

Key words. X-rays: galaxies: clusters - galaxies: clusters: general - cosmology: theory - turbulence

\section{Introduction}

In hierarchical structure formation scenarios clusters grow via accretion and merging of smaller subclumps. Gas accreting onto clusters of galaxies has bulk velocities of about $v=$ $1900(T / 6.7 \mathrm{keV})^{0.52} \mathrm{~km} \mathrm{~s}^{-1}$ at $1 \mathrm{Mpc}$ (e.g. Miniati et al. 2000), where $T$ is the mean X-ray temperature of the intracluster medium (ICM). This velocity is comparable to the expected sound speed of $1000-1500 \mathrm{~km} \mathrm{~s}^{-1}$ of the ICM. Accretion flows through filaments and sheets are highly asymmetric and produce complex patterns which can survive for long time-scales in the ICM (Miniati et al. 2000). Simulations by Norman \& Bryan (1999) predict that the turbulent pressure in the ICM can account for up to $20 \%$ of the thermal pressure. We thus expect some measurable effects of turbulence in the ICM of clusters of galaxies.

Concerning X-ray data, Inogamov \& Sunyaev (2003) propose a study of spectral line profiles as a useful diagnostic tool of turbulent flows in the ICM which could be measured with the future ASTRO-E2 satellite. Vogt \& Enßlin (2003) propose the application of Faraday Rotation measures to test turbulence in the ICM, and claim that for a few clusters a Kolmogorov spectrum seems to be plausible.

In the present investigation we show that turbulence in the ICM can be probed directly with pressure maps provided by the XMM-Newton satellite as a result of its high sensitivity and excellent spectral capabilities.

* Based on observations with XMM-Newton, an ESA Science Mission with instruments and contributions directly funded by ESA Member States and the USA (NASA).
Section 2 summarizes the basic phenomena related to turbulent flows. In Sect. 3 we give a simple analytic treatment of projection effects introduced through observation. In Sect. 4 we present the observational data and describe how our X-ray temperature and pressure maps are constructed. Based on the direct comparsion of local temperature and density measurements, we give in Sect. 5 some arguments that their observed fluctuations appear to be almost adiabatic. The same statistical analysis also suggests the absence of pronounced contact discontinuities and strong shocks. These observations provide a baseline consistent with the presence of a turbulent flow. Therefore, we study in detail in Sect. 6 the measured pressure spectrum in Fouriertransformed $k$ space and discuss its interpretation in Sect. 7. For all computations a flat geometry and a Hubble constant of $H_{0}=50 \mathrm{~km} \mathrm{~s}^{-1} \mathrm{Mpc}^{-1}$ are used. We assume a distance of $139 \mathrm{Mpc}$ to the Coma cluster so that 1 arcmin corresponds to about $40 \mathrm{kpc}$.

\section{Phenomenology of isotropic turbulence}

Traditionally, the phenomenology of isotropic turbulence is based either on second-order velocity statistics (Kolmogorov 1941) or on their Fourier-transformed counterparts (Oboukhov 1941). The velocity energy spectrum

$E_{v}(k)=C_{\mathrm{K}} \epsilon^{2 / 3} k^{-5 / 3}$

with the non-dimensional Kolmogorov constant $C_{\mathrm{K}}$, can be obtained for the inertial scale range from simple dimensional arguments (e.g., Lesieur 1997). In Eq. (1), $E_{v}(k)$ is the kinetic energy of fluctuations per unit mass and wavenumber $k$ (physical units $\mathrm{m}^{3} \mathrm{~s}^{-2}$ ), and $\epsilon$, in units of kinetic energy per unit mass 
Table 1. Spectrum $E_{v}(k)$ in the inertial range for different fluids, Mach numbers $\mathcal{M}$, and magnetic fields $B$.

\begin{tabular}{llll}
\hline \hline Turbulence & $\mathcal{M}$ & $B$-field & $E_{v}(k)$ \\
\hline$[1]$ & $<1$ & $B=0$ & $k^{-5 / 3}$ \\
{$[2]$} & $>1$ & $B=0$ & $k^{-6 / 3}$ \\
{$[3]$} & $<1$ & $B \neq 0$ & $k^{-5 / 3}$ \\
{$[4]$} & $>1$ & $B \neq 0$ & $k^{-3 / 2 \ldots-9 / 3}$ \\
\hline
\end{tabular}

[1] Kolmogorov (1941); Oboukhov (1941); [2] Burgers (1974); [3] Goldreich \& Sridhar (1995); [4] Cho \& Lazarian (2002); Vestuto et al. (2003).

and time $\left(\mathrm{m}^{2} \mathrm{~s}^{-3}\right)$, is the rate of kinetic energy transport from large to small scales. Equation (1) fundamentally stems from the assumption that, based on dimensional arguments, a turbulent eddy on a scale $\lambda \sim k^{-1}$, decays on a "turnover" timescale $\tau=\lambda / v(k)=\left[k^{3} E_{v}(k)\right]^{-1 / 2}$ (Oboukhov 1941). Since the kinetic energy associated with fluctuations over a scale $k^{-1}$ is $k E_{v}(k)$, in steady state regime the rate of energy transport across different scales is $\epsilon=k E_{v} / \tau$ which leads to Eq. (1).

In the inertial scale range where Eq. (1) applies, turbulence develops without being affected by boundaries, external forces, or viscosity. Here, the fluctuating quantities are assumed to be statistically invariant under translation (homogeneity) and rotation (isotropy). Table 1 summarizes some of the results from theoretical studies and numerical simulations, which suggest that Kolmogorov/Oboukhov-like spectra emerge in an inertial scale range under quite general conditions.

While all these studies are based on the analysis of velocity fluctuations, Oboukhov (1949) and Batchelor (1951) showed that gas pressure fluctuations also obey a scaling law (e.g., Lesieur 1997, Chap. VI),

$$
E_{P}(k)=C_{P} \epsilon^{4 / 3} k^{-7 / 3}
$$

where $C_{P}$ is a non-dimensional constant, and $E_{P}(k)$ has the units of kinetic energy per unit mass squared and is normalized to unit wavenumber $k$ (physical units $\mathrm{m}^{5} \mathrm{~s}^{-4}$ ). We note that the slope of the spectrum of the pressure is steeper than the spectrum of the velocity $\left(P \sim v^{2}\right)$. As for the velocity spectrum, we also expect that in general the exact slope of the pressure spectrum depends on whether or not the fluid is supersonic, and whether or not magnetic fields are present. The pressure spectrum thus appears as an excellent and powerful diagnostic tool of turbulent ICM flows. In addition, this approach appears quite attractive because pressure fluctuations can already be measured from high resolution X-ray data.

Establishing the presence of a turbulent ICM implies testing whether or not there is a scale-free inertial range in the pressure spectrum with a slope similar to the Kolmogorov/Oboukhov case. It is of further interest to measure the location of certain characteristic scales, such as the spectral break which corresponds to the scale where the kinetic energy is initially injected into the ICM, as well as the smallest scale where the corresponding energy is finally dissipated into the ICM. It is, however, not yet clear whether there is also energy dissipation within the inertial range caused by the development of randomly distributed weak shocks (Burgers turbulence, Table 1).

\section{Projection effects}

X-ray observations measure, after filtering and normalization (Sect. 6), a projection of the actual three-dimensional pressure fluctuations, $\delta P / P(\boldsymbol{r})$, on the two-dimensional celestial sphere. Note that the application of normalized instead of absolute quantities modifies the physical units of the structure function and power spectrum introduced in Sect. 2. Our analysis concentrates on scales which are small compared to the cluster core radius $r_{\mathrm{c}}$. As larger scales of order $r_{\mathrm{c}}$ are approached, the global cluster profile starts to be probed. This will be taken into account in the treatment (see Sect. 6). Thus, we start by decomposing the fluctuations into Fourier modes,

$\frac{\delta P}{P}(\boldsymbol{r})=\frac{1}{(2 \pi)^{3}} \int \mathrm{d}^{3} \boldsymbol{k} \frac{\delta P}{P}(\boldsymbol{k}) \mathrm{e}^{-\mathrm{i} \boldsymbol{k} \cdot \boldsymbol{r}}$.

The small-angle approximation allows us to regard the cluster region as composed of coplanar layers, with a pressure distribution assumed to be homogeneous and isotropic within each layer. We deliberately choose an X-ray energy band which is almost free of the temperature dependence of emissivity (see Sect. 4). A two-dimensional pressure field can thus be constructed using $\boldsymbol{r}=(\boldsymbol{R}, z)$,

$\frac{\delta P}{P}(\boldsymbol{R}) \sim \int \mathrm{d} z \frac{\delta P}{P}(\boldsymbol{r})\left[1+\frac{\left(z-z_{0}\right)^{2}}{r_{\mathrm{c}}^{2}}\right]^{-3 \beta}$,

which weights the pressure field of each layer with its emissivity, as obtained from a $\beta$ model of the cluster gas density (squared). In Eq. (4), $z_{0}$ is the distance between the observer and the cluster centre along the line-of-sight (LOS), and $\boldsymbol{R}$ a two-dimensional vector on the sky.

An illustration of the weighting scheme is shown for the Coma cluster in Fig. 1. Here, the contours give the percentage of surface brightness contributed by ICM gas within a distance $\left|z-z_{0}\right|$ from the plane through the cluster centre and perpendicular to the $z$ direction, as obtained by the integral of the squared $\beta$ profile along the $z$ direction. A comparison of the upper and lower panels of Fig. 1 reveals that the angular dependence of the general profile of the Coma cluster is imprinted on large scales, of order $>200 \mathrm{kpc}$, whereas on smaller scales the structure of Coma appears quite homogeneous in each layer, so that only a unique profile along the $z$ direction is seen. In the inner region, weighting can thus be approximated by taking into account only the variation of the density along the $z$ direction and neglecting the angular dependence as assumed in Eq. (4). For clusters with small core radii the approximation is less valid and the projection should be performed numerically.

A direct consequence of Eq. (4) or more complicated projection schemes is the invariance of relations between fluctuating quantities under geometric projections. This is illustrated for the adiabatic relation between temperature and density, $T \sim n^{\gamma-1}$, used in Sect. 5 to classify the fluctuations. Its differential version

$\frac{\delta T}{T}(\boldsymbol{r})=(\gamma-1) \frac{\delta n}{n}(\boldsymbol{r})$. 

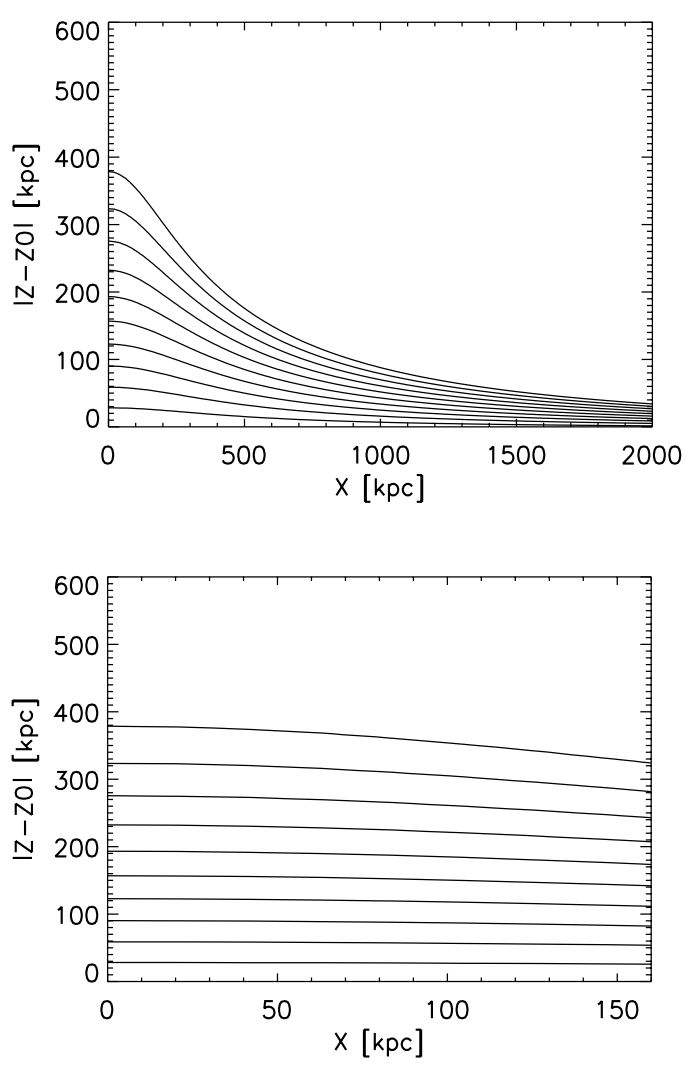

Fig. 1. Angular profile of the weight function ( $\beta$ profile squared) integrated along the $z$ direction for various integration lengths. The lowest/highest contours indicate $10 / 100 \%$ contributions, and the intermediate lines the contributions in steps of $10 \%$. The upper panel shows the contributions on cluster scales and the lower panel the contributions on the maximum scales of the observed turbulence structures (see Sect. 7). For the brightness distribution we assume a $\beta$ profile with the parameter values $\beta=0.75$ and $r_{\mathrm{c}}=420 \mathrm{kpc}$.

defines the (adiabatic) fluctuations. The projected temperature and density fluctuations are given by Eq. (4) replacing $P$ with $T$ and $n$. We further replace $\left[1+\left(z-z_{0}\right)^{2} / r_{\mathrm{c}}^{2}\right]^{-3 \beta}$ by $W(\boldsymbol{r})$, i.e., a general weighting function which describes the geometric projection process. If the weighting $W(\boldsymbol{r})$ were the same for $T$ and $n$, then we could write

$$
\begin{aligned}
\frac{\delta T}{T}(\boldsymbol{R}) & =\text { const. } \int \mathrm{d} z \frac{\delta T}{T}(\boldsymbol{r}) W(\boldsymbol{r}) \\
& =(\gamma-1) \text { const. } \int \mathrm{d} z \frac{\delta n}{n}(\boldsymbol{r}) W(\boldsymbol{r}) \\
& =(\gamma-1) \frac{\delta n}{n}(\boldsymbol{R}) .
\end{aligned}
$$

However, two-dimensional (projected) temperature maps, $T(\boldsymbol{R})$, resulting from X-ray observations, are $n^{2}(\boldsymbol{r})$-weighted averages along the LOS of the three-dimensional field $T(\boldsymbol{r})$. On the contrary, projected squared densities, $n^{2}(\boldsymbol{R})$, are obtained by a simple geometric mean without any weighting. For this more realistic case we thus have

$\frac{\delta T(\boldsymbol{R})}{T(\boldsymbol{R})}=\frac{\int \mathrm{d} z \delta T(\boldsymbol{r}) n^{2}(\boldsymbol{r})}{\int \mathrm{d} z T(\boldsymbol{r}) n^{2}(\boldsymbol{r})}=\frac{\gamma-1}{2} \frac{\int \mathrm{d} z T(\boldsymbol{r}) \delta n^{2}(\boldsymbol{r})}{\int \mathrm{d} z T(\boldsymbol{r}) n^{2}(\boldsymbol{r})}$,

where $\delta n^{2}=2 n \delta n$ and Eq. (5) have been used. For the assumed central core region of the cluster we now have the advantageous situation that $T(\boldsymbol{r})$ assumes the role of an almost constant weighting function for the density, and we can show that retaining only first order terms, Eq. (6) simplifies to

$$
\frac{\delta T(\boldsymbol{R})}{T(\boldsymbol{R})} \approx \frac{\gamma-1}{2} \frac{\int \mathrm{d} z \delta n^{2}(\boldsymbol{r})}{\int \mathrm{d} z n^{2}(\boldsymbol{r})}=\frac{\gamma-1}{2} \frac{\delta n^{2}(\boldsymbol{R})}{n^{2}(\boldsymbol{R})} .
$$

Equation (7) is the observational counterpart of Eq. (5) and will be used in Sect. 5 to constrain possible types of pressure fluctuations measured in the centre of the Coma cluster.

After cross-correlating projected fluctuation measures, we now proceed with projecting pressure spectra. We still assume that the central cluster region, to which our analyses are restricted, is small enough to be approximated by a set of coplanar, homogeneous, and isotropic layers, yielding a unique pressure profile along the $z$ direction. We want to take advantage of the invariance property of a Gaussian profile under Fourier transformation by replacing the $\beta$ model by a Gaussian with variance $r_{\mathrm{c}}^{2} / 3 \beta$. The approximation is better than $5 \%$ for $r<r_{\mathrm{c}}$ and allows us to regard the convolution of the pressure field with the gas density profile in Eq. (4) along the $z$ direction as a transformation $\delta P / P(k) \rightarrow \delta P / P(k) \exp \left(-k_{z}^{2} r_{\mathrm{c}}^{2} / 6 \beta\right)$. With the standard relations between two and three-dimensional power spectra (e.g., Peacock 1999, Sect. 18.1) we obtain the simple expression

$\mathcal{P}_{2 \mathrm{D}}(K)=\frac{1}{\pi} \int_{0}^{\infty} \mathrm{d} k_{z} \mathcal{P}_{3 \mathrm{D}}\left(\sqrt{k_{z}^{2}+K^{2}}\right) \exp \left(-\frac{k_{z}^{2} r_{\mathrm{c}}^{2}}{3 \beta}\right)$,

where the integration extends over the wavenumber $k_{z}$ along the $z$ direction. The wavenumbers in two and in three dimensions are $K$ and $k$, respectively. Note that $K=2 \pi / \Theta$, with $\Theta$ being the angular scale of the projected fluctuation, has the physical units $\mathrm{rad}^{-1}$, but can be readily transformed to metric scales using the distance to the cluster. Equation (8) shows that adding three-dimensional fluctuations along the $z$ direction leads to an exponentially damped two-dimensional spectrum. Damping is larger for smaller modes because relatively more fluctuations are added along the $z$ direction.

The relations between power spectra and pressure spectra in two and three-dimensions can be obtained from the condition that the sum over both statistics must give the same total energy,

$$
\begin{aligned}
& \frac{1}{(2 \pi)^{2}} \int_{0}^{\infty} 2 \pi K \mathrm{~d} K \mathcal{P}_{2 \mathrm{D}}(K)=\int_{0}^{\infty} E_{2 \mathrm{D}}(K) \mathrm{d} K, \\
& \frac{1}{(2 \pi)^{3}} \int_{0}^{\infty} 4 \pi k^{2} \mathrm{~d} k \mathcal{P}_{3 \mathrm{D}}(k)=\int_{0}^{\infty} E_{3 \mathrm{D}}(k) \mathrm{d} k,
\end{aligned}
$$

which yields the following conversions:

$\mathcal{P}_{2 \mathrm{D}}(K)=2 \pi K^{-1} \quad E_{2 \mathrm{D}}(K)$,

$\mathcal{P}_{3 \mathrm{D}}(k)=2 \pi^{2} k^{-2} \quad E_{3 \mathrm{D}}(k)$.

This gives the projection of the pressure spectrum

$$
E_{2 \mathrm{D}}(K)=K \int_{0}^{\infty} \frac{\mathrm{d} k_{z}}{k_{z}^{2}+K^{2}} E_{3 \mathrm{D}}\left(\sqrt{k_{z}^{2}+K^{2}}\right) \mathrm{e}^{-\frac{k_{z}^{2} r_{\mathrm{c}}^{2}}{3 \beta}},
$$

where $E_{3 \mathrm{D}}(\cdot)$ can be identified with the spectrum Eq. (2) after including appropriate upper and lower integration limits. Note 
Table 2. Summary of XMM-Newton observations.

\begin{tabular}{lllccccc}
\hline \hline & Name of & & \multicolumn{3}{c}{ pn-Camera observing times (ks) } & Orbit & Frametime \\
\multicolumn{1}{c}{ Date } & observation & RA(2000) & Dec(2000) & Planned & Effective & & ms \\
\hline 2000 May 29 & Coma center & 125946.7 & 275700 & 15.0 & 12.8 & 86 & 199 \\
2000 June 21 & Coma 1 & 125647.7 & 272407 & 25.0 & 21.1 & 98 & 73 \\
2000 June 11 & Coma 2 & 125742.5 & 274338 & 25.0 & 43.8 & 93 & 199 \\
2000 June 27 & Coma 3 & 125832.2 & 272412 & 25.0 & 11.0 & 101 & 73 \\
2000 June 23 & Coma 4 & 130004.6 & 273124 & 25.0 & 5.4 & 99 & 199 \\
2000 May 29 & Coma 5 & 125927.5 & 274653 & 20.0 & 9.9 & 86 & 199 \\
2000 June 12 & Coma 6 & 125850.0 & 275852 & 20.0 & 12.4 & 93 & 199 \\
2000 Dec. 10 & Coma 7 & 125727.7 & 280841 & 25.0 & 18.6 & 184 & 199 \\
2000 Dec. 10/11 & Coma 8 & 130125.6 & 274353 & 26.0 & 14.3 & 184 & 199 \\
2000 June 11/12 & Coma 9 & 130032.7 & 275659 & 20.0 & 14.7 & 93 & 199 \\
2000 June 22 & Coma 10 & 125938.4 & 280740 & 20.0 & 15.4 & 98 & 199 \\
2000 June 24 & Coma 11 & 125836.5 & 282356 & 25.0 & 11.5 & 99 & 199 \\
2002 June 5/6 & Coma 12 & 130150.2 & 280928 & 25.0 & 9.6 & 456 & 199 \\
2002 June 7/8 & Coma 13 & 130036.5 & 282515 & 25.0 & 20.0 & 457 & 199 \\
2000 June 22 & Coma 0 (bkg) & 130137.0 & 271952 & 30.0 & 12.8 & 98 & 199 \\
2001 Dec. 4/5 & Coma cal & 125946.6 & 275700 & 25.0 & 17.4 & 364 & 73 \\
\hline
\end{tabular}

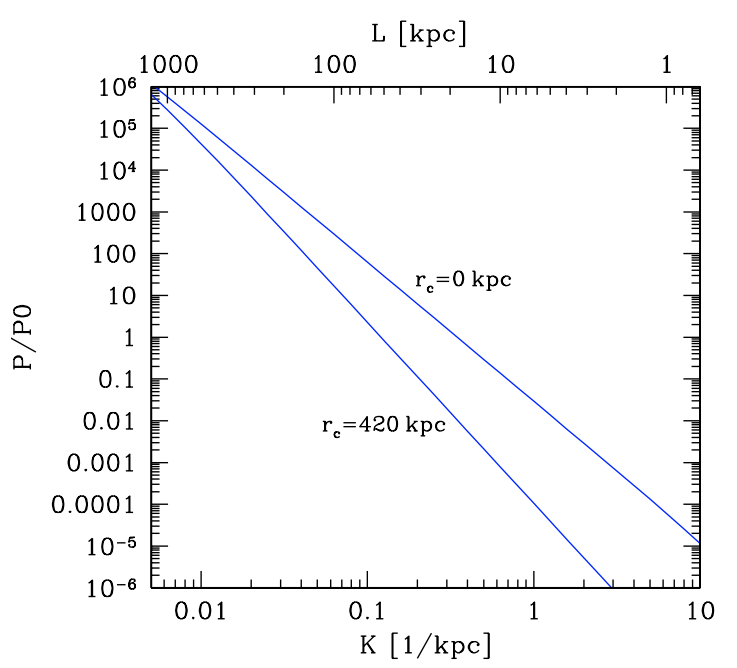

Fig. 2. Pressure power spectra with an intrinsic slope of $n=-7 / 3$ as expected for a Kolmogorov/Oboukhov turbulence $\left(r_{\mathrm{c}}=0\right)$, and its projection $(420 \mathrm{kpc})$, as seen along the $z$ direction through the central plane of a cluster with a core radius $\left(r_{\mathrm{c}}=420 \mathrm{kpc}\right)$ and slope parameter $(\beta=0.75)$ as measured for the Coma cluster. In order to illustrate the projection effects over a large scale range, we did not introduce any characteristic scale which limits the spectra at large and small scales.

that the decline of $E_{3 \mathrm{D}}(k)$ and thus of $\mathcal{P}_{3 \mathrm{D}}(k)$ outside the inertial range may not be sharp, and instead better described by smooth characteristic shape functions (see, e.g., Fritsch et al. 1980). Figure 2 gives a quantitative impression of the projection effects on a power spectrum computed with Eq. (8) for the Coma cluster with distance $139 \mathrm{Mpc}, \beta=0.75$, and core radius $r_{\mathrm{c}}=420 \mathrm{kpc}$. For illustration, no $K$ limits of the inertial range are introduced. For comparison we also plot the $k_{z} r_{\mathrm{c}} \ll 1$ case where no corrections for projection are necessary. The intrinsic slope of the pressure spectrum is $n=-7 / 3$. Note that the power spectrum of the pressure fluctuations is damped at $60 \mathrm{kpc}$ by a factor of about 28 . The observation of pressure fluctuations along the $z$ direction through the cluster thus damps their amplitudes by a factor $\sqrt{28} \approx 5$.3. However, cluster-wide fluctuations on Mpc-scales would appear almost undamped.

Further observational effects are related to the measurement errors of the pressure and the global pressure profile and can be illustrated best with the observed spectrum of the cluster pressure distribution (Sect. 6).

\section{The Coma mosaic}

In this paper we use the performance verification observations of the Coma cluster obtained with the EPIC-pn instrument on board of XMM-Newton (Jansen et al. 2001). Previous reports of these observations were given by Briel et al. (2001), Arnaud et al. (2001), Neumann et al. (2001, 2003) and Finoguenov et al. (2004a, for point-like sources). This work includes all datasets obtained to date, as described in detail by Finoguenov et al. (2004a, see also Table 2). While most of the pointings have been obtained in Extended Full Frame Mode (Frametime $199 \mathrm{~ms}$ in Table 2), three observations were conducted in Full Frame Mode (Frametime $73 \mathrm{~ms}$ ).

All observations have been reprocessed using the latest version of the XMM reduction pipeline (XMMSAS 5.4.1), which yields an astrometry to better than 1 arcsec. Although the Coma data are public, for some (Coma-10 and Coma-0) of the early observations of the performance verification phase no complete Observational Data Files (ODF) had been produced by standard processing, and a special preprocessing (XMMSAS task odffix) was done on such pn exposures at MPE by Michael Freyberg. As a result, a few Coma pointings are not yet publicly available, which precluded us from using MOS data. The vignetting correction, crucial for obtaining reliable source 

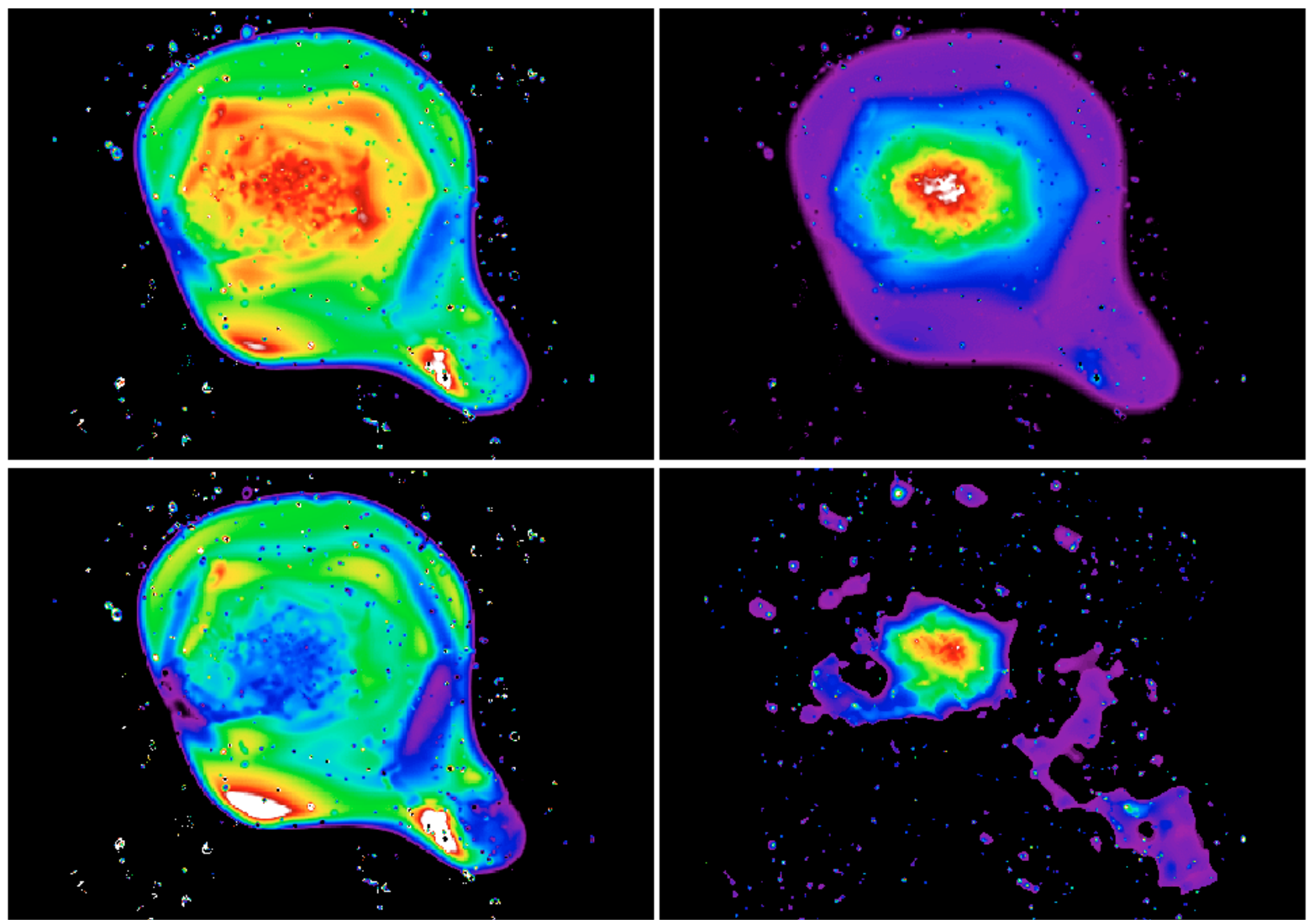

Fig. 3. Projected temperature map (upper left), pressure map (upper right), entropy map (lower left) and image substructure (residual) map as revealed by small-scales in the wavelet decomposition (lower right). The maps are obtained from spectral hardness ratios and surface brightness data and smoothed with a wavelet filter of the Coma cluster. Each map covers an area of $93 \times 93 \mathrm{arcmin}^{2}$.

characteristics over a wide region, is performed using the latest calibration (Lumb et al. 2003). Two pointings at the Coma centre were used in that calibration, in a way requiring that the same sky pixels yield the same flux between the two observations. The level of the emission was not used in the calibration, so it could be analyzed further. The RMS fluctuations of the comparison of two Coma fields is within $2 \%$, which will affect the apparent pressure fluctuations studied here on the $1 \%$ level, much lower than the observed $10 \%$ amplitudes.

The images were extracted separately for each pointing, along with the corresponding exposure maps. We select pn events with PATTERN $<5$ and (FLAG\&0xc3b0809) $=0$, which in addition to FLAG $=0$ events includes events in the rows close to gaps and bad pixels; however, it excludes the columns with offset energy. This event selection results in a better spatial coverage of the cluster, but at a somewhat compromised energy resolution, which is sufficient for the broadband imaging.

Our final results are derived from a spectral analysis where only the FLAG $=0$ events were retained. For background subtraction we used the similarly screened and selected events from the background accumulation of Andrew Read (Read \& Ponman 2003) and also subtracted out-of-time events as a background, using products from the SAS task epchain. This subtraction is important as some pointings (see FrameTime $73 \mathrm{~ms}$ in Table 2) are performed in the Full Frame Mode.
To provide an overview of the structure of the ICM of the Coma cluster, we show in Figs. 3 and 4 the temperature, pressure, and entropy maps, as well as maps of the small-scale surface brightness structure. These maps use hardness ratios in the $0.8-2 \mathrm{keV}$ and $2-7.5 \mathrm{keV}$ bands, calibrated for the measurement of temperature, as a substitute for the temperature determined directly from the spectral analysis. The projected entropy $(S)$ and projected pressure $(P)$ maps are derived from the projected temperature, $T$, and surface brightness, $\Sigma$, through the relations $S=T \Sigma^{-1 / 3}$ and $P=T \Sigma^{1 / 2}$, respectively. The maps are constructed from composite wavelet filtered images to suppress the large scale background. The details of the analysis based on surface brightness and hardness ratio maps and the rationale of the use of wavelet filtering is described in detail in previous publications (Briel et al. 2004; Finoguenov et al. 2004b; Henry et al. 2004).

The substructures seen in these maps suggest turbulent-like fluctuations. The pressure maps are of special importance because they clearly show fluctuations which are not contaminated by contact discontinuities (see Sect. 5). For a quantitative study of the significance of these fluctuations we thus performed direct fits to the spectral X-ray data.

In Fig. 5 we show the temperature, entropy and pressure maps, based on the temperature and emission measure obtained through direct spectral fitting, using several grids to define the region of spectral extraction. Only the $16 \times 16$ grids with a pixel size $40 \times 40 \operatorname{arcsec}^{2}, 120 \times 120 \operatorname{arcsec}^{2}, 260 \times 260 \operatorname{arcsec}^{2}$ are 


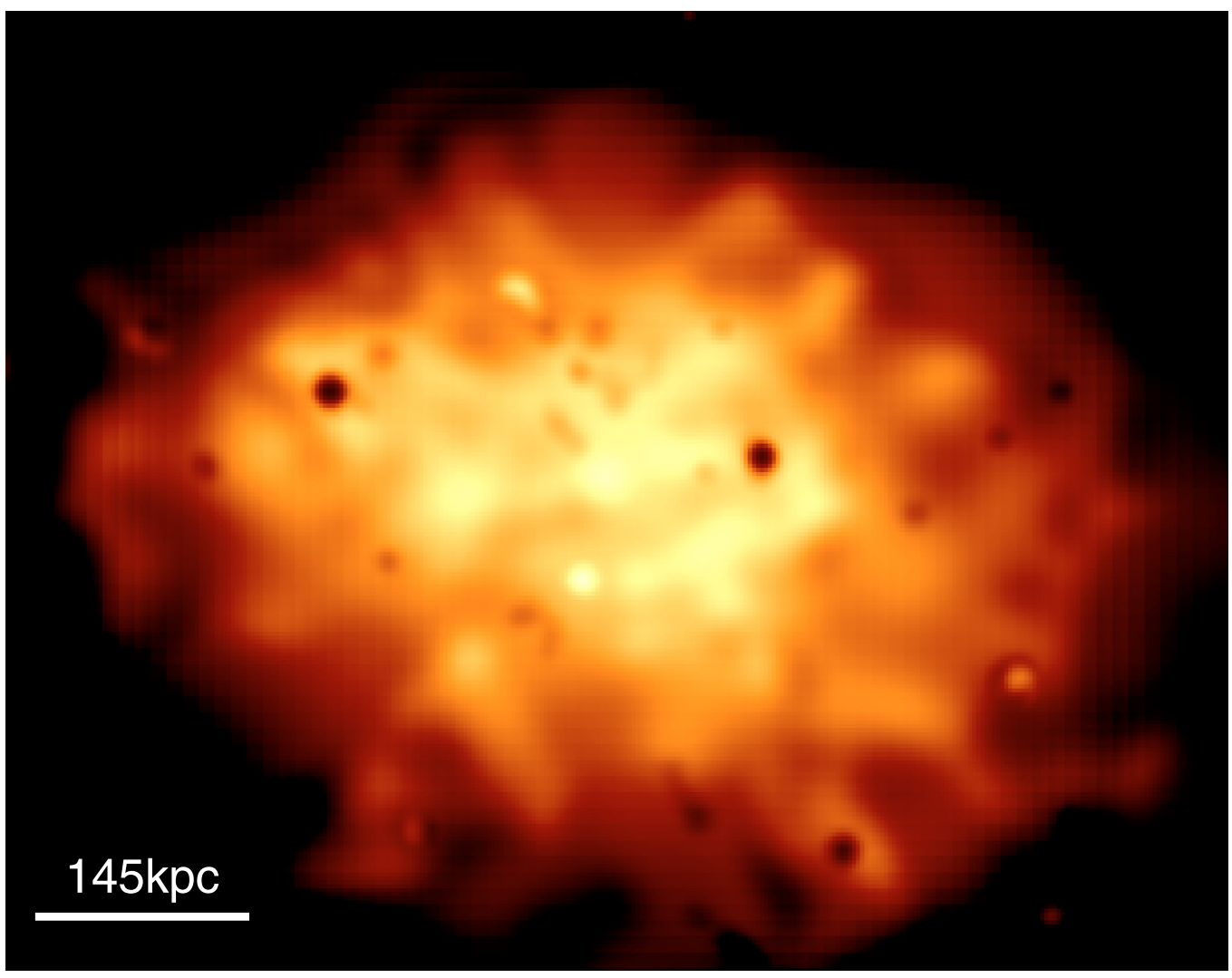

Fig. 4. Detailed view of the projected pressure distribution of the central region of the Coma cluster. The $145 \mathrm{kpc}$ scale corresponds to the largest size of the turbulent eddies indicated by the pressure spectrum (Sect. 7). The smallest turbulent eddies have scales of around $20 \mathrm{kpc}$. On smaller scales the number of photons used for the spectral analysis is too low for reliable pressure measurements.

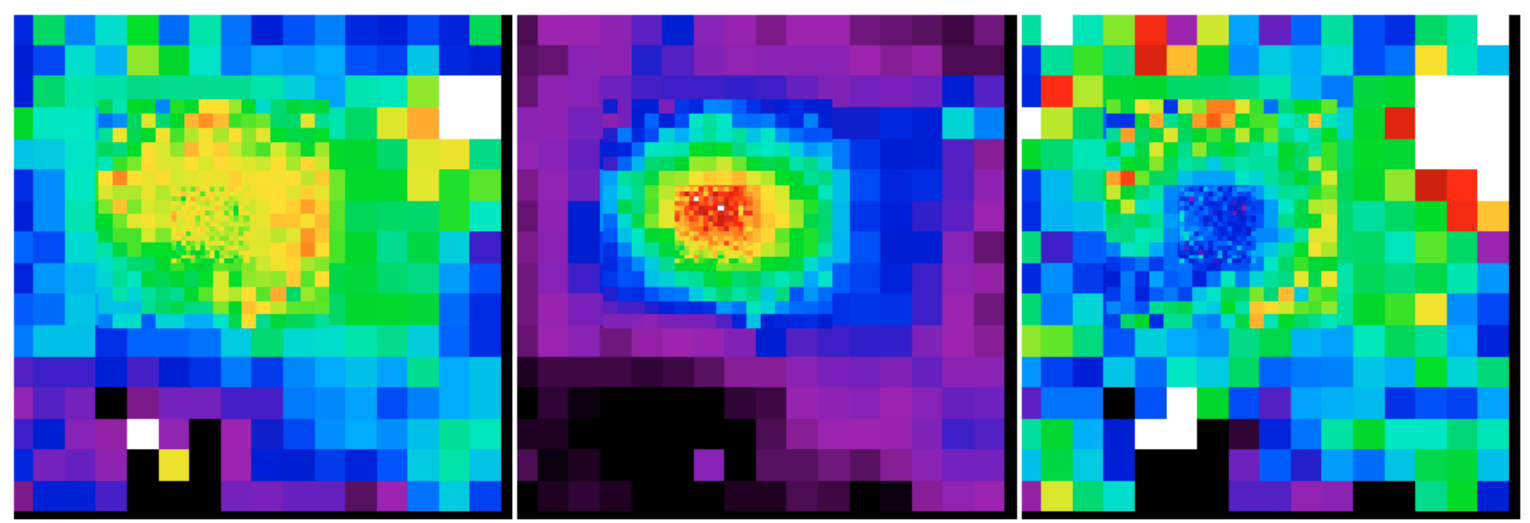

Fig. 5. Nested grids of temperature (left panel), pressure (middle panel), and entropy (right panel) measurements. Each map covers an area of $69.3 \times 69.3 \operatorname{arcmin}^{2}$.

shown. This figure also illustrates the relative positioning of the grids. Each binning involves a mixing of various spectral components. Therefore, a decision has to be made on which of the components the spectral analyses should be performed. We have chosen to put our interest on the hotter component, and so have used the $1-7.9 \mathrm{keV}$ energy band for spectral fitting. Fine grids, with a pixel size of $40 \times 40 \operatorname{arcsec}^{2}$ and lower, located in the central region, do not suffer that much from temperature mixing, but they do suffer from small number statistics. So, for those we used the $0.5-7.9 \mathrm{keV}$ band. A detailed check has shown that for a similar location in the Coma cluster all grids yield similar temperature estimates, which supports our choice of energy bands. The selection of the grid resolution was performed to yield at least 5000 counts per pixel. The total number of counts available for the analysis in the Coma observation reaches two million counts in the $0.5-2 \mathrm{keV}$ band and a similar amount in the harder band $(2-7.9 \mathrm{keV})$.

\section{General character of the fluctuations}

In order to obtain more information about the type of fluctuations seen in Figs. 3-5, we performed a pixel-by-pixel crosscomparison of temperature and density gradients. 


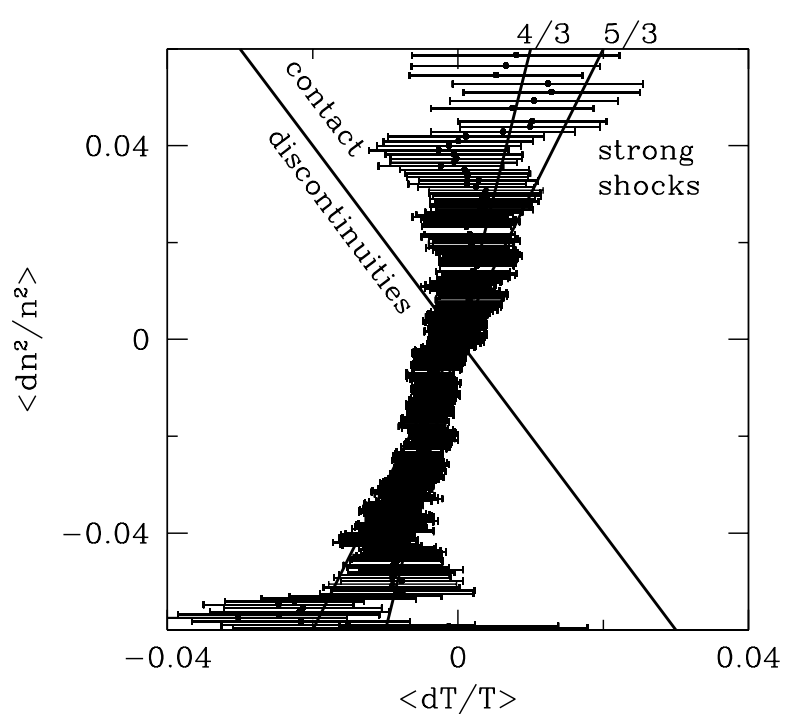

Fig. 6. Correlation between relative fluctuations of density squared $\left(n^{2}\right)$ and temperature $(T)$, and their $1 \sigma$ errors. The two thick lines represent the adiabatic exponent $\gamma=5 / 3$ (monoatomic ideal gas) and $\gamma=4 / 3$ which gives a good representation of the data. Due to the sliding window method used, neighbouring data points are correlated.

Figure 6 shows the correlation between the gradients of the projected X-ray temperature and the gradients of the projected squared gas density as obtained for the $40 \times 40 \operatorname{arcsec}^{2}$ pixel grid. We concentrate on this specific grid because it is mainly restricted to the core region of the Coma cluster and has sufficiently high signal-to-noise X-ray spectra at a comparatively small angular resolution.

The relative fluctuations are determined for each pixel of the temperature and density map by averaging the gradients over its four nearest neighbour pixels. The averaged gradients are obtained for density and temperature maps and can be compared in a point-wise manner. However, the individual fluctuations are large ( $10 \%$ level) because they include also the measurement errors. Therefore, an additional binning with $\mathrm{d} n^{2} / n^{2}=0.1$ and a continuous sliding of this bin along the density axis is necessary to see a clear trend. The error bars are the $1 \sigma$ fluctuations of the mean obtained for each bin.

For the classification of the fluctuations, we show in Fig. 6 model expectations obtained with Eq. (7). The line labeled $\gamma=5 / 3$ corresponds to a monoatomic ideal gas. For contact discontinuities local pressure equilibrium leads to $\delta n / n=$ $-\delta T / T$. Apparently, the $\gamma=4 / 3$ line gives a better representation than what is expected for the $\gamma=5 / 3$ case. This could be due to a contamination by contact discontinuities.

Since the gradients are measured on scales of $27 \mathrm{kpc}$, which are small compared to the cluster core radius of $r_{\mathrm{c}}=420 \mathrm{kpc}$, we expect them to be sensitive probes of local substructure fluctuations and not significantly affected by the global cluster profile. We nevertheless tested this approximately with Monte Carlo simulations where the gradients of $n^{2}$ are computed with an isothermal $\beta$ model of the Coma cluster and a gas adiabatic equation of state. The gradients are determined in the same way as the empirical data and added to the adiabatic density fluctuations. We found that large-scale gradients in the density field broaden the scaling relation, but without introducing a bias in the determination of $\gamma$. In fact, we have verified this for different values of the adiabatic exponent $\gamma$.

The observed temperature and density maps are also tested for possible correlations between $T$ and $n^{2}$ introduced by the large-scale distribution of the ICM. The radial profiles are obtained by averaging temperatures and densities in concentric rings with a width of $50 \mathrm{kpc}$. Whereas $n^{2}$ shows a significant decrease of $27 \pm 2$ percent between the cluster center and $300 \mathrm{kpc}$, the temperature decreases by only $3 \pm 2$ percent relative to the central value of $7.34 \pm 0.13 \mathrm{keV}$. The observed temperature gradients thus appear with the same size as the errors and can thus be neglected as a possible second-order effect. Within this approximation, no correlations between temperature and density fluctuations are introduced by the global cluster profile.

To conclude, Fig. 6 suggests a positive correlation between temperature and density gradients which is not related to the large-scale distribution of the ICM. The gradients occupy different regions than contact discontinuities and strong shocks. The data are actually quite close to the expected adiabatic case. In order to find out whether or not such fluctuations are organized as in a turbulent regime, we study in the following the statistics of the spatial pressure fluctuations.

\section{Power spectrum of spatial pressure fluctuations}

The first step in our (standard) power spectrum analysis is the determination of the global pressure profile $\bar{P}(\boldsymbol{R})$ from the observed 2-dimensional pressure map $P(\boldsymbol{R})$, in order to get the residual local pressure fluctuations, $\delta P / P(\boldsymbol{R})=P(\boldsymbol{R}) / \bar{P}(\boldsymbol{R})-1$. The second step is the determination of the Fourier power spectrum of $\delta P / P(\boldsymbol{R})$, corrected for the errors of the pressure measurements (shot-noise subtraction), and normalized to unit number of Fourier modes and to unit area in $K$ space. The resulting projected spectrum $\mathcal{P}_{2 \mathrm{D}}(K)$ has the physical units $\mathrm{kpc}^{2}$. In the following example, the pressure is measured in a regular grid of $32 \times 32$ cells, each with $20 \times 20 \operatorname{arcsec}^{2}$. This grid covers the central core region of Coma up to $431 \mathrm{kpc}$ and has the fundamental mode $K=2 \pi / \lambda=0.0146 \mathrm{kpc}^{-1}$. The results obtained with the other three grids are given at the end of this section.

The global pressure profile $\bar{P}(\boldsymbol{R})$ is obtained from a lowpassband Fourier-filter applied to $P(\boldsymbol{R})$ with a filter scale of $150 \mathrm{kpc}$, which leaves the global cluster profile above this scale almost unchanged. To illustrate the effect of the filter, we show in Fig. 7 the power spectrum (marked "P") obtained from a direct Fourier-transformation of $P(\boldsymbol{R})$. On scales between 20 and $40 \mathrm{kpc}$, the spectrum has a flat plateau-like distribution which is determined by the temperature and density errors (shot-noise, see below). Between 40 and $125 \mathrm{kpc}$ the spectrum increases significantly above the shot-noise level. This is the spectrum of the substructures seen in Figs. 3 to 5 . Beyond $125-150 \mathrm{kpc}$, the spectrum abruptly increases due to the global pressure profile of the Coma cluster.

A similar increase is also seen in the spectrum marked "Wavelet" which is obtained alternatively from a waveletfiltered pressure map. For the wavelet decomposition we used 


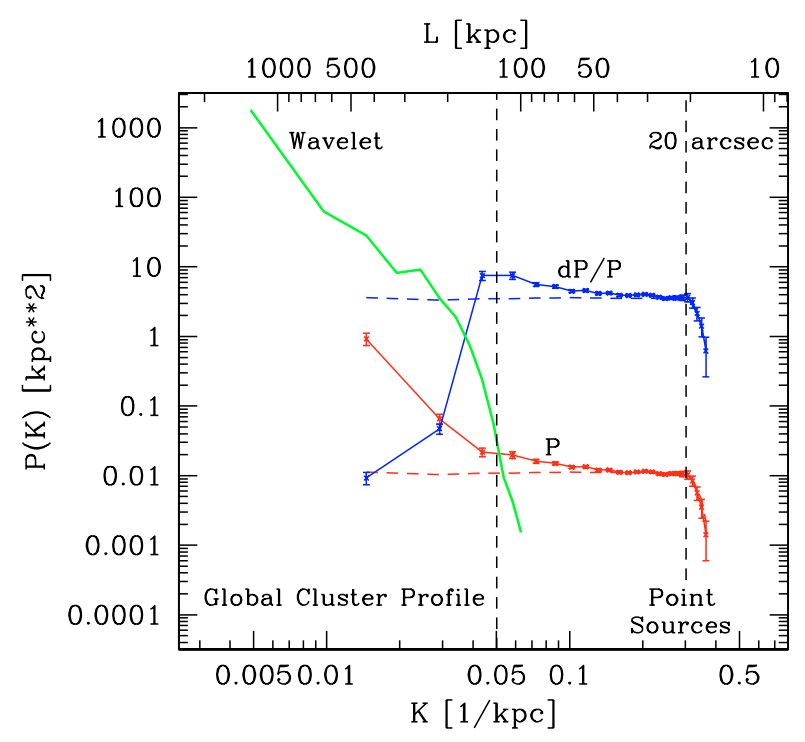

Fig. 7. Projected power spectra of different angular pressure distributions from the $20 \times 20 \operatorname{arcsec}^{2}$ grid. Lower thin continuous line: raw spectrum including shot-noise, substructure, and cluster profile (P). Upper thin continuous line: spectrum from the normalized pressure distribution $(\mathrm{d} P / P)$. Thick continuous line: spectrum of the global cluster pressure profile as determined with the wavelet transform. Dashed horizontal lines: shot-noise levels computed from measured pressure errors. Dashed vertical lines: characteristic scales.

the algorithm of Vikhlinin et al. (1998) and computed the spectrum from the wavelet reconstruction of the $30 \times 30 \operatorname{arcsec}^{2}$ map with the lowest angular resolution. The wavelet algorithm performs a self-adjusting noise suppression so that almost no significant shot-noise occurs in the spectrum of the global pressure profile. The $20 \times 20 \operatorname{arcsec}^{2}$ grid does not cover the complete cluster area and is thus not optimal for the proper sampling of the global cluster profile. Therefore, the similarity of the "P" and "Wavelet" spectra is not very good on large scales. However, grids with larger bin sizes cover larger scales and give a very good agreement with the "Wavelet" profile (see below). For the following analyses we thus use the Fourier lowpass filter with a filter scale of $150 \mathrm{kpc}$ to determine $\bar{P}(\boldsymbol{R})$ for all four grids.

The histogram of the resulting $\delta P / P(\boldsymbol{R})$ is shown in Fig. 8 . Their distribution appears quite consistent with a Gaussian random field (KS-probability of $90 \%$ ) with zero mean and a standard deviation of 15 percent (including shot-noise) on a pixel scale of $13.5 \mathrm{kpc}$. The $\delta P / P(\boldsymbol{R})$ field can thus completely be summarized by a power spectrum. The corresponding power spectral densities are marked by " $\mathrm{d} P / P$ " in Fig. 7. The spectrum shows the expected drop beyond $150 \mathrm{kpc}$. A similar drop at scales below $20 \mathrm{kpc}$ marks the resolution limit as given by the pixelation (see "Point Sources"). These two cutoff scales limit the range of the power spectrum of the substructures.

The effect of temperature and density measurement errors is seen in the power spectra as an almost scaleindependent shot-noise level which must be subtracted from the " $\mathrm{d} P / P$ "-spectrum (dashed horizontal lines in Fig. 7). For the determination of the shot-noise, we first determine at each grid point the local relative pressure error $\sigma_{P}$, using $T$ and its

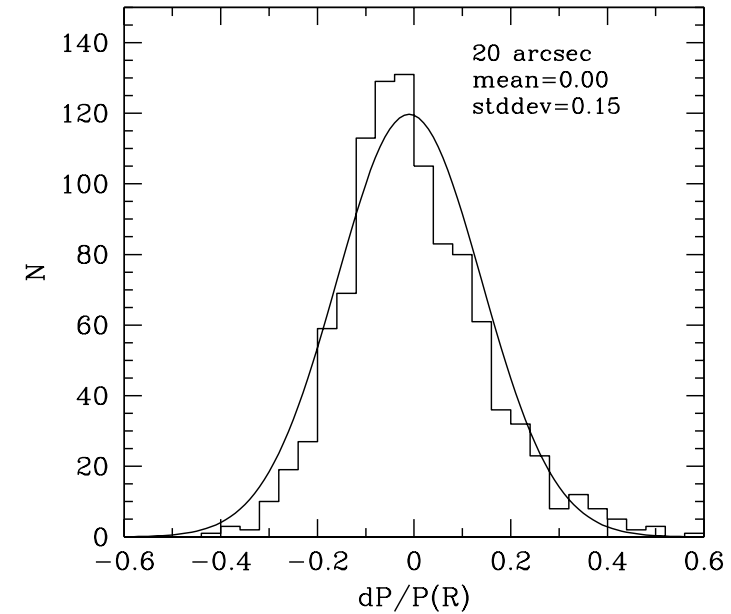

Fig. 8. Comparison of a Gaussian profile with the histogram of projected pressure contrasts $\delta P / P=[P(\boldsymbol{R}) / \bar{P}]-1$ in configuration space for a pixel size of $20 \times 20 \operatorname{arcsec}^{2}$ which corresponds to $13.5 \times$ $13.5 \mathrm{kpc}^{2}$. The mean over all fluctuation is almost zero and the $1 \sigma$ standard variation $\sigma_{\delta P / P}=0.15$.

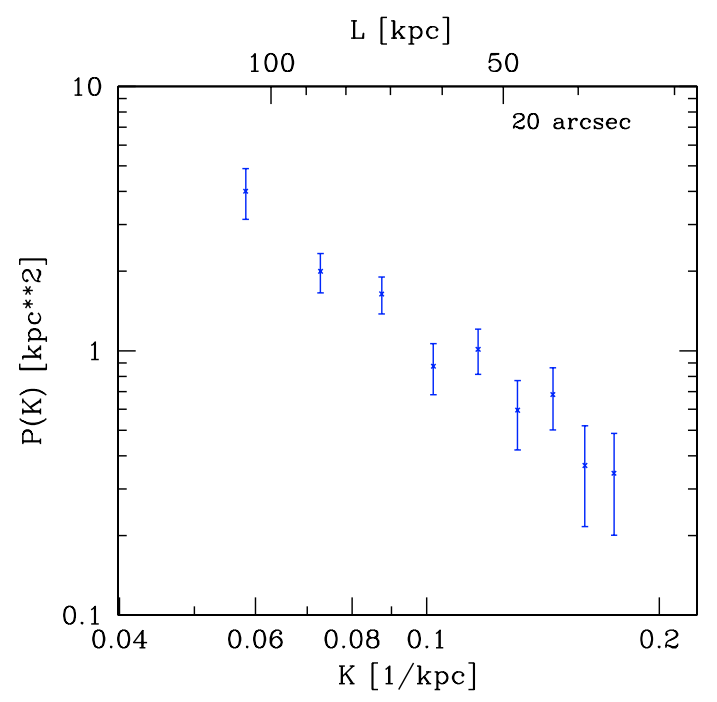

Fig. 9. Projected shot-noise subtracted power spectral densities, $\mathcal{P}_{2 \mathrm{D}}(K)$, of the pressure fluctuations, and their $1 \sigma$ errors, obtained from the $20 \times 20 \operatorname{arcsec}^{2}$ grid.

error $\sigma_{T}$ as well as $n^{2}$ and its error $\sigma_{n^{2}}$, as obtained from the local X-ray spectral fit. We then draw at each grid point a random value for the local relative pressure error from a Gaussian distribution with zero mean and standard deviation $\sigma_{P}$. After performing this randomization for all grid points, one realisation of a random map is generated with fluctuations solely caused by measurement errors. For the determination of the shot-noise we averaged the power spectra of 100 random realisations.

The $1 \sigma$ error bars shown in Fig. 7 are also determined from the variances of the spectra obtained from randomized maps of the measurement errors. The errors are lower limits because they are obtained from unstructured pressure maps. Unfortunately, much larger effort is needed to improve these estimates, for example, with a set of hydrodynamical cluster simulations. 


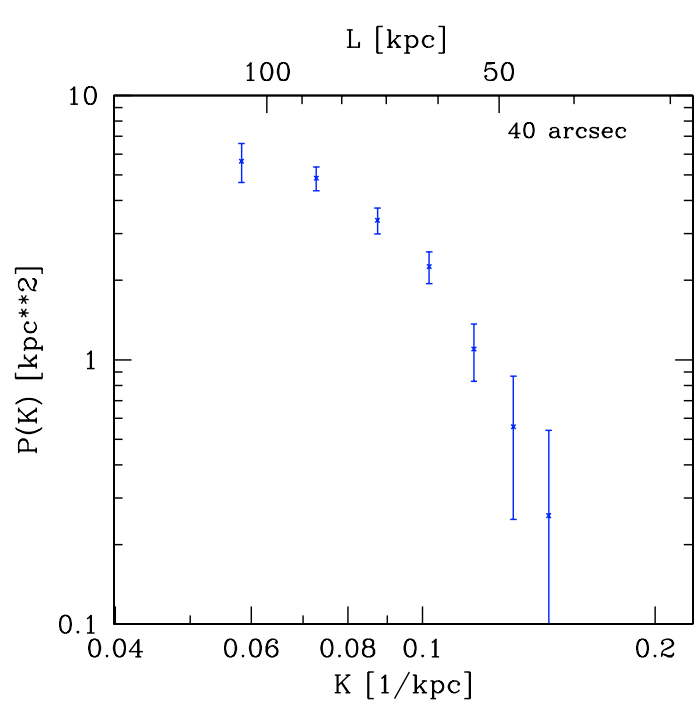

Fig. 10. Projected shotnoise subtracted power spectral densities, $\mathcal{P}_{2 \mathrm{D}}(K)$, as in Fig. 9 for the $40 \times 40 \operatorname{arcsec}^{2}$ grid.

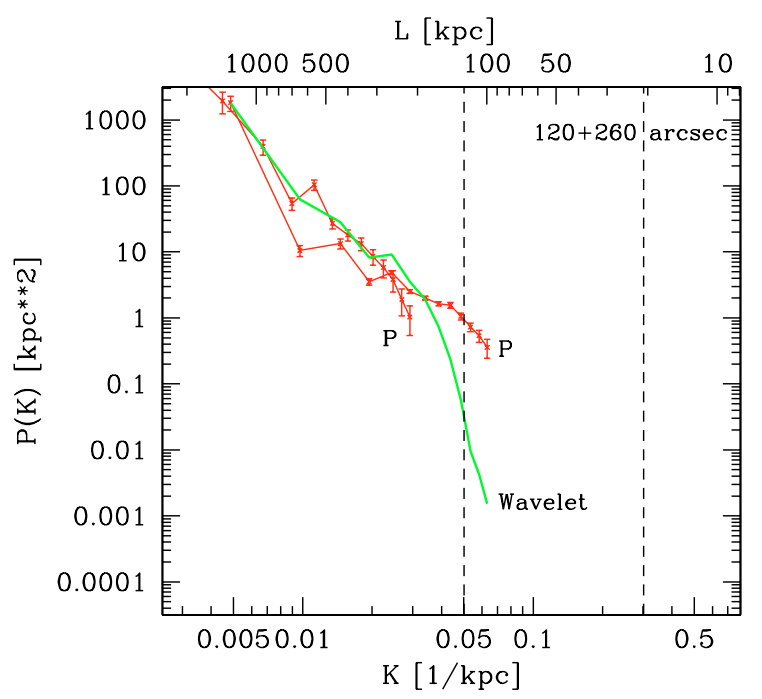

Fig. 11. Projected power spectral densities as in Fig. 7 for the $120 \times$ $260 \operatorname{arcsec}^{2}$ and the $260 \times 120 \operatorname{arcsec}^{2}$ grid. The spectra basically follow the global cluster pressure profile.

Figure 9 shows the power spectrum of $\delta P / P(\boldsymbol{R})$ after shot-noise subtraction. This spectrum can be compared via Eq. (8) with theoretical 3-dimensional power spectra or structure functions.

The same analysis performed on the $40 \times 40 \operatorname{arcsec}^{2}$ grid gives the power spectrum shown in Fig. 10. The errors are smaller compared to the results obtained with the $20 \times 20 \operatorname{arcsec}^{2}$ grid. We attribute this to the higher signal-tonoise X-ray spectra obtained with the larger pixels. The spectral shape appears somewhat more curved and steeper than the spectrum obtained with the smaller grid. The $120 \times 120 \operatorname{arcsec}^{2}$ and $260 \times 260 \operatorname{arcsec}^{2}$ grids mainly sample the global cluster profile. The resulting "P"-spectra shown in Fig. 11 follow the profile obtained with the wavelet-filtered pressure distribution. These spectra do not have any significant fluctuation power on scales below $100 \mathrm{kpc}$ so that we do not show the corresponding "d $P / P$ "-spectra.

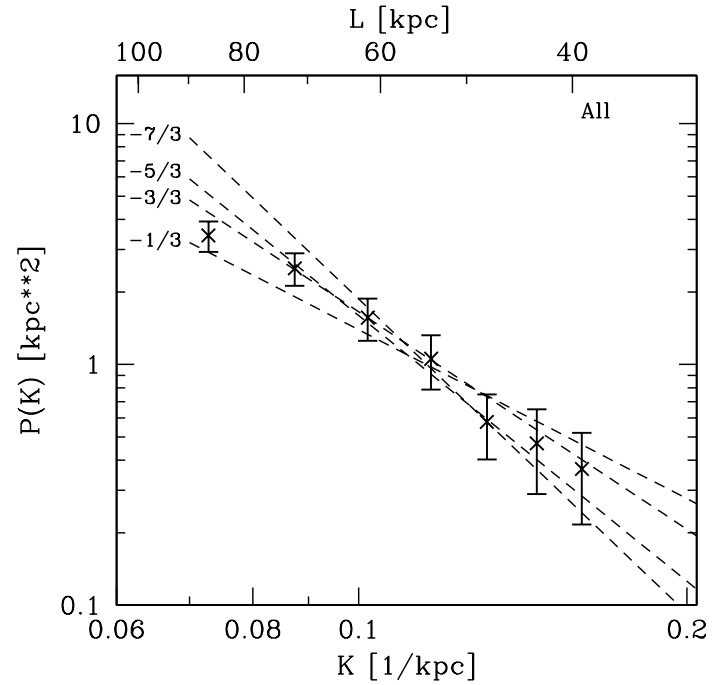

Fig. 12. Observed projected shot-noise-subtracted power spectral densities (dots with $1 \sigma$ error bars) as obtained for the $20 \times 20 \operatorname{arcsec}^{2}$ and $40 \times 40 \operatorname{arcsec}^{2}$ grids, compared with model predictions (dashed lines).

The differences seen in the power spectral densities shown in Figs. 9 and 10 are caused by the chosen center, bin size, and total size of the sample grid. This sample variance can be reduced by averaging the spectral densities measured at the same $K$-values. The average is meaningful because all spectral densities - although determined in $K$ bins with different sizes (fundamental modes) - are normalized to the same unit volume of $1 / \mathrm{kpc}^{2}$ in $K$ space. The random errors of the spectral densities which do not refer to sample variance are not reduced because the two power spectra cannot be regarded as completely statistically independent. We regard the spectrum shown in Fig. 12 as the final result of the power spectrum analysis.

\section{Discussion}

The present investigation aims to detect turbulence in the ICM of the Coma cluster using the pressure spectrum. Under certain approximations, one also expects a scale-invariant spectrum of temperature fluctuations to be a probe of ICM turbulence (e.g. Lesieur 1997). However, this relies on the assumption that temperature behaves as a passive scalar. Once this has been verified empirically, the almost uniform distribution of the temperature over scales $\gg r_{\mathrm{c}}$ allows a cleaner distinction between small-scale turbulent substructures and the large-scale cluster profile. However, in reality temperature maps are affected by cold fronts and other contact discontinuities which contaminate the diagnostic maps. On the other hand, pressure maps have a clear relation to velocity $\left(P=\rho v^{2}\right)$ and are not significantly contaminated by contact discontinuities. Therefore, we regard pressure as a more direct probe of ICM turbulence.

The mosaic of XMM-Newton observations is well-suited for the detection of turbulence in the Coma cluster because it allows a better geometric discrimination between pressure variations originating from the overall cluster profile, and substructure superimposed onto it. This transition occurs at about $150 \mathrm{kpc}$. 
The measured temperature and density gradients (Fig. 6) suggest that the substructures have an adiabatic exponent of $\gamma \approx 4 / 3$, which is close to the adiabatic case of an ideal monoatomic gas. On the other hand, contact discontinuities and strong shocks seem to be less likely in the core region, consistent with hydrodynamical simulations (Miniati et al. 2000; Miniati 2003). In addition, the statistics of the residual pressure fluctuations appear quite Gaussian (Fig. 8) emphasising their random nature. Their Fourier power spectrum thus completely summarizes the fluctuating pressure field and can be used to obtain observational evidence for the presence of turbulent flows which are characterized by a Kolmogorov/Oboukhovlike spectrum.

Figure 12 shows the combined power spectrum of the Coma cluster on scales between 40 and $90 \mathrm{kpc}$. A scaleinvariant range of the spectrum is indicated and suggests the detection of an inertial range of a turbulent ICM. Theoretical three-dimensional power spectra,

$$
\begin{aligned}
\mathcal{P}_{3 \mathrm{D}}(k) & =2 \pi^{2} k^{-2} E_{3 \mathrm{D}}(k)=2 \pi^{2} C_{\mathrm{P}} \epsilon^{4 / 3} k^{n-2} \\
& =2 \pi^{2} C k^{n-2},
\end{aligned}
$$

are transformed with Eq. (8) into their two-dimensional counterparts (dashed lines in Fig. 12). From the comparison of observed and theoretical spectra we see that on scales between 40 and $60 \mathrm{kpc}$, the observed power spectrum has a slope between $n=-7 / 3$ and $-5 / 3$. This slope corresponds to the spectral slope of the Fourier-transformed Kolmogorov/Oboukhov structure function (Eq. (2)). On scales between 60 and $90 \mathrm{kpc}$ the spectrum bends towards smaller slopes between $n=-5 / 3$ and $-1 / 3$. The normalization constants range from $C=$ $C_{\mathrm{P}} \epsilon^{4 / 3}=0.0063 \mathrm{kpc}^{-4 / 3}$ for $n=-7 / 3$, to $C=0.470 \mathrm{kpc}^{2 / 3}$ for $n=-1 / 3$. An absolute calibration of the pressure used in our analyses is in preparation and will give a direct estimate of $\epsilon$. In this respect it would be interesting to compare this rate of kinetic energy transport with the observed X-ray luminosity.

The power spectrum shown in Fig. 10 allows a first estimation of the location of the characteristic scale where the spectrum sharply drops towards larger scales. This scale is at approximately $\lambda_{\mathrm{i}} \approx 100 \mathrm{kpc}$ and should be regarded as a lower limit because of possible contaminations by the global cluster profile. This scale should also roughly correspond to the injection scale (e.g., Lesieur 1997), and it is similar to estimates for the impact parameter of merging clusters based on kinematics and tidal torque-based arguments (e.g., Sarazin 2002).

The integral of the power spectrum (Eq. (10)) is expected to give important information about the energy deposited in turbulent motion. For the scale range between 40 and $90 \mathrm{kpc}$, the slope and amplitude parameters derived above yield relative contributions of the turbulent pressure to the thermal pressure between 7.4 percent for $n=-7 / 3$, and 6.6 percent for $n=-1 / 3$. The largest possible contributions are obtained with the $n=-7 / 3$ spectrum. Therefore, we computed for this spectrum the relative contribution for different minimum scales, i.e., lower integration limits of the inertial range (dissipation scale), and maximum scales, i.e., upper integration limits (injection scale).

Figure 13 shows that for a fixed turbulence spectrum the relative contribution is mainly determined by the value of the

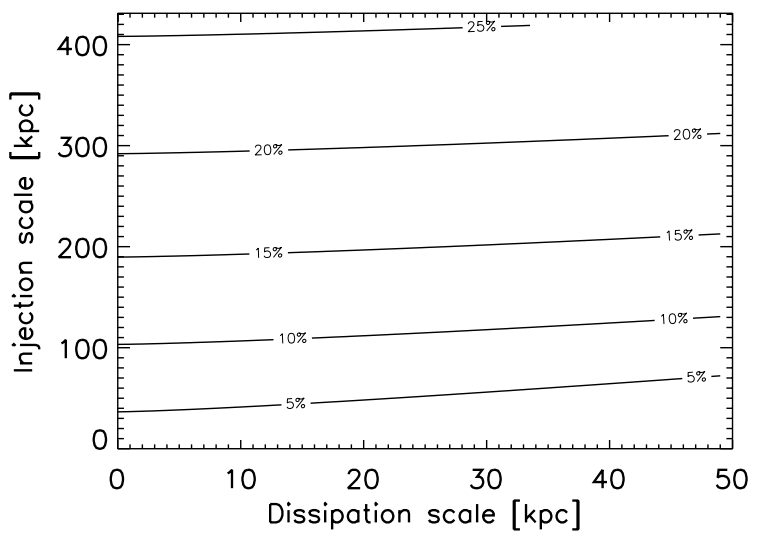

Fig. 13. Contribution of the turbulent pressure to the total thermal pressure (contour lines of equal percentage) for a Kolmogorov/Oboukhov spectrum with the slope $n=-7 / 3$ and the amplitude $C=C_{\mathrm{P}} \epsilon^{4 / 3}=0.0063 \mathrm{kpc}^{-4 / 3}$. The power spectrum is integrated between the injection and the dissipation scale.

injection scale $\lambda_{\mathrm{i}}$. We do not see any turbulent eddies of the size of the core radius of $420 \mathrm{pc}$ in Fig. 4 which could have erroneously been subtracted by the Fourier low-pass filter although they still might be present, but are difficult to discriminate from the global cluster profile. Therefore, the relative contribution of the turbulent pressure to the thermal pressure should be smaller than 25 percent. If we take the indication for a turnover in the power spectrum shown in Fig. 10 at $\lambda_{\mathrm{i}}=100 \mathrm{kpc}$ as the injection scale, then we would get a lower limit of about 10 percent. The simulations of Norman \& Bryan (1999) suggest additional support by turbulent pressure of about 20 percent, averaged over the cluster ( 5 to 35 percent between core and virial radius), which is apparently of the same order as the present observational limit. However, further study is definitely required in order to establish how the observational quantities relate to the simulation results.

For the observed turbulent ICM we can now estimate the kinematic viscosity by assuming that magnetic fields have a negligible effect (see below). For a turbulent flow the Reynolds number of the global fluid $\mathfrak{R}$ measured at the injection scale $\lambda_{\mathrm{i}}$, and the Reynolds number $\mathfrak{R}_{\mathrm{d}}$ measured at the dissipation scale $\lambda_{\mathrm{d}}$ are related by $\mathfrak{R} / \mathfrak{R}_{\mathrm{d}}=\left(\lambda_{\mathrm{i}} / \lambda_{\mathrm{d}}\right)^{4 / 3}$. The power spectra do not show any tendency to decrease at $\lambda=30 \mathrm{kpc}$ (Fig. 9). Therefore, $\lambda_{\mathrm{d}}$ is smaller than $30 \mathrm{kpc}$. In the following we assume a fiducial value of $\lambda_{\mathrm{d}}=10 \mathrm{kpc}$. The turbulent flow in the central region of Coma can thus be characterized by $\mathfrak{R} / \mathfrak{R}_{\mathrm{d}}>20$. Reynolds numbers at dissipation scales are expected to be above unity so that $\mathfrak{R}$ will have values in excess of 20. Although this estimate is rather conservative, this is the best that can be obtained by direct observations at the moment.

For the viscosity we further need the velocity at the injection scale. This number can be obtained from hydrodynamical simulations (Miniati et al., in preparation), which typically give for an $8 \mathrm{keV}$ cluster a dispersion turbulent velocity of $v_{\lambda_{\mathrm{i}}}=250 \mathrm{~km} \mathrm{~s}^{-1}$ on scales of $\lambda_{\mathrm{i}} \approx 100 \mathrm{kpc}$. This provides a quite reliable upper limit to the kinematic viscosity of

$v<3 \times 10^{29}\left(\frac{v_{\lambda_{\mathrm{i}}}}{250 \mathrm{~km} \mathrm{~s}^{-1}}\right)\left(\frac{\lambda_{\mathrm{i}}}{100 \mathrm{kpc}}\right)\left(\frac{\mathfrak{R}}{20}\right)^{-1}\left[\frac{\mathrm{cm}^{2}}{\mathrm{~s}}\right]$. 
Note that the coherence lengths of magnetic fields in the cores of galaxy clusters as obtained from Faraday Rotation measurements are about 5-10 kpc (e.g., Taylor \& Perley 1993) and thus below the scale range covered by the present data. Therefore, we regard the upper limit (Eq. (16)) as not significantly affected by magnetic fields.

Fabian et al. (2003, see also Reynolds et al. 2004) assume laminar flow of the ICM around the radio galaxy NGC 1275 with $v_{\lambda}=700 \mathrm{~km} \mathrm{~s}^{-1}$ in the centre of the Perseus cluster on $\lambda=14 \mathrm{kpc}$ scales. From the laminar appearance of the filaments they assume that the effective Reynolds number is less than 1000 so that they estimate the lower limit $v>4 \times 10^{27}\left[\frac{\mathrm{cm}^{2}}{\mathrm{~s}}\right]$. The upper limit obtained from a turbulent regime and the lower limit obtained from a laminar regime can be used to estimate the range $10-30 \mathrm{kpc}$ where the transition from a turbulent to a laminar flow could occur. This corresponds to a dissipation scale of the ICM in the same range. A remark of caution is, however, necessary here, because we compare two different situations (merger driven turbulence versus AGN driven turbulence, and a bulk ICM in Coma versus condensed warm HII-gas in the NGC 1275 halo), and it is not fully clear in how far they are comparable.

Shibata et al. (2001) determined the 2-point angular correlation function of hardness ratios as a measure of the temperature fluctuations detected with ASCA over an area of 19 square degrees in the Virgo cluster. A significant excess of the correlation amplitude is found at $300 \mathrm{kpc}$. They interpreted the random temperature fluctuations in Virgo-North as local heating of infalling galaxy groups.

Future investigations should measure the pressure spectrum of the Coma cluster more accurately down to $5 \mathrm{kpc}$ so that the combination with the present measurements would give information about the ICM in the Coma cluster from 5-2800 kpc. This could give us tight constraints on the type of gas turbulence, its energy content, the importance of magnetic fields, and on the viscosity of the ICM.

Acknowledgements. The XMM-Newton project is supported by the Bundesministerium für Bildung und Forschung/Deutsches Zentrum für Luft- und Raumfahrt (BMFT/DLR), the Max-Planck Society and the Heidenhain-Stiftung, and also by PPARC, CEA, CNES, and ASI. We would like to thank Eugene Churazov and Alex Lazarian for helpful discussions. We also thank the anonymous referee for useful comments. A.F. acknowledges receiving the Max-Plank-Gesellschaft Fellowship and support from the Verbundforschung grant 50 OR 0207 of the DLR. F.M. was partially supported by the Research and Training Network "The Physics of the Intergalactic Medium" set up by the European Community under the contract HPRN-CT2000-00126 RG29185. P.S. acknowledges support under the DLR grant No. 50 OR 970835.

\section{References}

Arnaud, M., Aghanim, N., Gastaud, R., et al. 2001, A\&A, 365, L67

Batchelor, G. K. 1951, Proc. Camb. Phil. Soc., 47, 359

Briel, U. G., Henry, J. P., Lumb, D. H., et al. 2001, A\&A, 365, L60

Briel, U. G., Finoguenov, A., \& Henry, J. P. 2004, A\&A, 426, 1

Burgers, J. M. 1974, The Nonlinear Diffusion Equation (Dordrecht: Reidel)

Cho, J., \& Lazarian, A. 2002, PhRvL, 88, 245001

Fabian, A. C., Sanders, J. S., Crawford, C. S., et al. 2003, MNRAS, 344, L48

Finoguenov, A., Briel, U. G., Henry, P. J., et al. 2004a, A\&A, accepted Finoguenov, A., Pietsch, W. N., Aschenbach, B. R., \& Miniati, F. 2004b, A\&A, 415, 415

Fritsch, U., Lesieur, M., \& Schertzer, D. 1980, J. Fluid Mech., 97, 181

Goldreich, P., \& Sridhar, H. 1995, ApJ, 438, 763

Henry, J. P., Finoguenov, A., \& Briel, U. G. 2004, ApJ, submitted

Inogamov, N. A., \& Sunyaev, R. A. 2003, AstL, 29, 791

Jansen, J., Lumb, D., Altieri, B., et al. 2001, A\&A, 365, L1

Kolmogorov, A. N. 1941, Dokl. Akad. Nauk SSSR, 30, 301

Lesieur, M. 1997, Turbulence in Fluids (Dordrecht: Kluwer Academic Publisher)

Lumb, D. H., Finoguenov, A., Saxton, R., et al. 2003, SPIE, 4851, 255

Miniati, F. 2003, MNRAS, 342, 1009

Miniati, F., Ryu, D., Kang, H., et al. 2000, ApJ, 542, 608

Neumann, D. M., Arnaud, M., Gastaud, R., et al. 2001, A\&A, 365, L74

Neumann, D. M., Lumb, D. H., Pratt, G. W., \& Briel, U. G. 2003, A\&A, 400, 811

Norman, M. L., \& Bryan, G. L. 1999, Lect. Not. Phys., 530, 106

Oboukhov, A. M. 1941, Dokl. Akad. Nauk SSSR, 32, 22

Oboukhov, A. M. 1949, Dokl. Akad. Nauk SSSR, 66, 17

Peacock, J. A. 1999, Cosmological Physics (Cambridge University Press)

Read, A. M., \& Ponman, T. J. 2003, A\&A, 409, 395

Reynolds, C. S., McKernan, B., Fabian, A. C., et al. 2004, MNRAS, submitted [arXiv:astro-ph/0402632]

Sarazin, G. 2002, in Merging Processes in Galaxy Clusters, ed. L. Feretti, I. M. Gioa, \& G. Giovannini, APSS Library (Dordrecht: Kluwer Academic Publishers), 272, 1

Shibata, R., Matsushita, K., Yamasaki, N. Y., et al. 2001, ApJ, 549, 228

Taylor, G. B., \& Perley, R. A. 1993, ApJ, 416, 554

Vestuto, J. G., Ostriker, E. C., \& Stone, J. M. 2003, ApJ, 590, 858

Vikhlinin, A., McNamara, B. R., Forman, W., et al. 1998, ApJ, 502, 558

Vogt, C., \& Enßlin, T. A. 2003, A\&A, 412, 373 